\title{
Investigation of the coupling between tunable split-ring resonators
}

\author{
Milo Baraclough, ${ }^{*}$ Ian R. Hooper, and William L. Barnes \\ Centre for Doctoral Training in Metamaterials, Department of Physics and Astronomy, University of Exeter, \\ Stocker Road, Exeter EX4 4QL, United Kingdom
}

(Received 13 June 2018; revised manuscript received 31 July 2018; published 28 August 2018)

\begin{abstract}
Passive resonant metamaterials are limited by the narrow-band nature of the resonances they support. Here we show that by incorporating an active component into the structure of the commonly used split-ring resonator it is possible to tune the resonance frequency of this type of metamaterial atom. We make use of this tunability to examine the interaction between two resonators, one passive and one active, as the resonance frequency of the active resonator is swept through that of the passive resonator. The resultant modes of this coupled system exhibit an anticrossing and, by changing the separation between, and relative orientation of, the split-ring resonators, we investigate how the magnetic and electric coupling terms change. We find that the relative orientation of the resonators significantly effects the strength of the coupling. Through both structural and active tuning we are able to alter the relative sizes and signs of the coupling terms. We hope that the nature of these changes will be of use to those designing large actively tunable metamaterial systems.
\end{abstract}

DOI: 10.1103/PhysRevB.98.085146

\section{INTRODUCTION}

The fabrication of structures that can achieve electromagnetic properties not found in nature has been an exciting topic of research for many years, perhaps beginning with Lamb's consideration of a wave with a backward group velocity in 1904 [1]. At the time it was thought that to achieve such a wave would not be possible due to the material properties that would be required. However, the explosion of interest in metamaterials in the 1990s, i.e., materials whose bulk properties rely on structure rather than chemistry, offers a new path to demonstrating Lamb's backwards wave, for example by making use of subwavelength arrays of rings and wires. While the field of metamaterials has continued to grow [2-4], one of the early components of metamaterials, the split-ring resonator (SRR), is still in common use as a "meta-atom." We use this meta-atom in the work reported here.

Split-ring resonators may be easily scaled in size so as to enable operation over a wide range of frequencies, and they have been studied from the low $\mathrm{GHz}$ up to visible frequencies [5-7]. Incident radiation polarized so that the magnetic field lies along the axis of the SRR induces both a circulating current in the ring and an electric field across the split [5], with corresponding electric and magnetic dipole moments that are indicated in Fig. 1. When many SRRs are arranged in an array, the individual SRRs may couple together in a manner analogous to classically coupled harmonic oscillators. This coupling has led to studies on the effects of the spacing

*mb504@exeter.ac.uk

Published by the American Physical Society under the terms of the Creative Commons Attribution 4.0 International license. Further distribution of this work must maintain attribution to the author(s) and the published article's title, journal citation, and DOI. and relative orientation of SRRs in one-, two-, and threedimensional arrays [8-16].

However, in common with many metamaterial elements, split-ring resonators have a limited frequency range over which they are resonant. While work has been undertaken to fabricate metamaterials with a broad-band response $[17,18]$, the structures used are still fixed at manufacture and sacrifice the ability to dynamically modify the operating frequency, an attribute that would be of considerable use in filter and antenna applications. More particularly, dynamical tuning would allow for functionality over a range of frequencies without sacrificing the benefits of selectivity offered by a narrow-band response. Such tuning has been achieved structurally $[19,20]$ by using the relationship between near-field interactions of the rings and their relative positions to tune the resonant response.

While the utility of active metamaterial elements for applications is clear, they can also be used as tools for investigating fundamental physics. For example, the use of metamaterial elements with active gain components enables loss to be compensated for, thereby allowing the investigation of paritytime symmetry breaking [21,22].

In this paper we investigate the coupling between an active tunable split-ring resonator and an adjacent passive SRR as a function of their separation and relative orientation. We show that, by altering these two parameters, the strength of the interaction between the SRRs can be altered dramatically, allowing a range of coupling regimes to be explored in a single system.

\section{ACTIVE TUNING}

The behavior of split-ring resonators is well understood, making them an ideal starting point for developing active meta-atoms. SRRs behave as resonant $L C$ circuits and, at their simplest, may be understood as a parallel plate capacitor (the split) connected in series with a single loop inductor (the ring). 


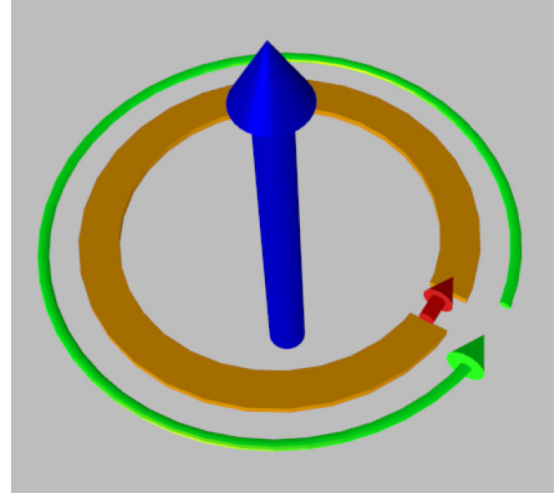

FIG. 1. Schematic to illustrate the dipole moments associated with a split-ring resonator. The magnetic (blue) and electric (red) dipole moments of a split-ring resonator are shown, along with the direction of the instantaneous current around the ring (green). Note that the electric dipole moment is localized around the split region where there is maximal charge accumulation, while the magnetic dipole moment passes through the center of the ring due to the circulating currents.

The fundamental resonance frequency of a split-ring resonator is given by Eq. (1), where $L$ is the effective inductance of the ring and $C$ is the effective capacitance [23],

$$
\omega_{0}=\frac{1}{\sqrt{L C}} .
$$

To make the response of the split-ring resonator tunable a variable capacitor (varactor) may be added [24]. A varactor consists of two layers of doped silicon, $P$ type and $N$ type (which are positively and negatively charged, respectively). At the boundary between these layers a depletion region develops. The thickness of this depletion region, and hence the capacitance of the device, can be altered by applying an external voltage; the higher the voltage, the more charge is driven across the depletion region, causing it to widen and the capacitance to fall.

By incorporating a varactor into a split-ring resonator (a schematic of the SRR showing the position of the varactor can be seen in Fig. 2) the total capacitance is altered since now the capacitance of the varactor has been added in series to the gap capacitance. Due to the reciprocal addition of capacitances in series, the smaller of the two contributions to the total capacitance dominates. Our design, with a narrow gap of 0.1 $\mathrm{mm}$ and a relatively thick ring width of $1 \mathrm{~mm}$, was chosen to maximize the capacitive over the inductive contribution to the resonance frequency, thereby increasing the tuning range of the SRR. We used an Infineon BB 857-02 V H7902 varactor, which allows the capacitance to be varied between $0.5 \pm 0.05 \mathrm{pF}$ at a bias of $28 \mathrm{~V}$, and $5 \pm 1 \mathrm{pF}$ at a bias of $0 \mathrm{~V}$ (taken from the manufacturer's specifications).

A coplanar waveguide, a type of rf transmission line, was chosen to excite the split-ring resonator. When a signal is transmitted along a CPW, electric fields oscillate between the central "signal" track and two grounded sheets on either side, marked A and G, respectively, in Fig. 2. There is often an additional ground plane on the underside of a CPW to further improve transmission by confining the fields, but this is omitted here and replaced instead with a pair of SRRs printed (one beneath each track to maintain symmetry with the $\mathrm{CPW}$ ) onto the underside of a substrate using standard PCB lithography and etching techniques. (Note that finite element modeling has been used to confirm that any interaction between the two rings is small and has a negligible effect on their resonant frequency. However, by placing an SRR beneath both tracks of the CPW the coupling between the SRRs and the radiation guided along the CPW is increased.) The SRRs are excited via the fields of the guided mode of the CPW, with the distance between the plane of the CPW and the plane containing the SRRs, and the separation of the pair of SRRs from the midline of the CPW, both determining the strength of the excitation. (Note: An offset of the SRR's position from the midline is required for maximum coupling to the guided mode due to the field curvature around the coplanar waveguide tracks. For a more detailed exploration of the interactions of SRRs and CPWs the reader is directed to Baena et al.'s work [25]). A fiberglass FR4 substrate with a relative permittivity of 4.5 , and coplanar waveguide dimensions of $t=5 \mathrm{~mm}$ and $s=0.5 \mathrm{~mm}$, were chosen to give a $50 \Omega$ impedance [26] (see right panel of Fig. 2). The use of a coplanar waveguide to excite the split-ring resonators allows easy access to the varactor, and a bias voltage to be applied without the soldered wires interfering with the exciting field.
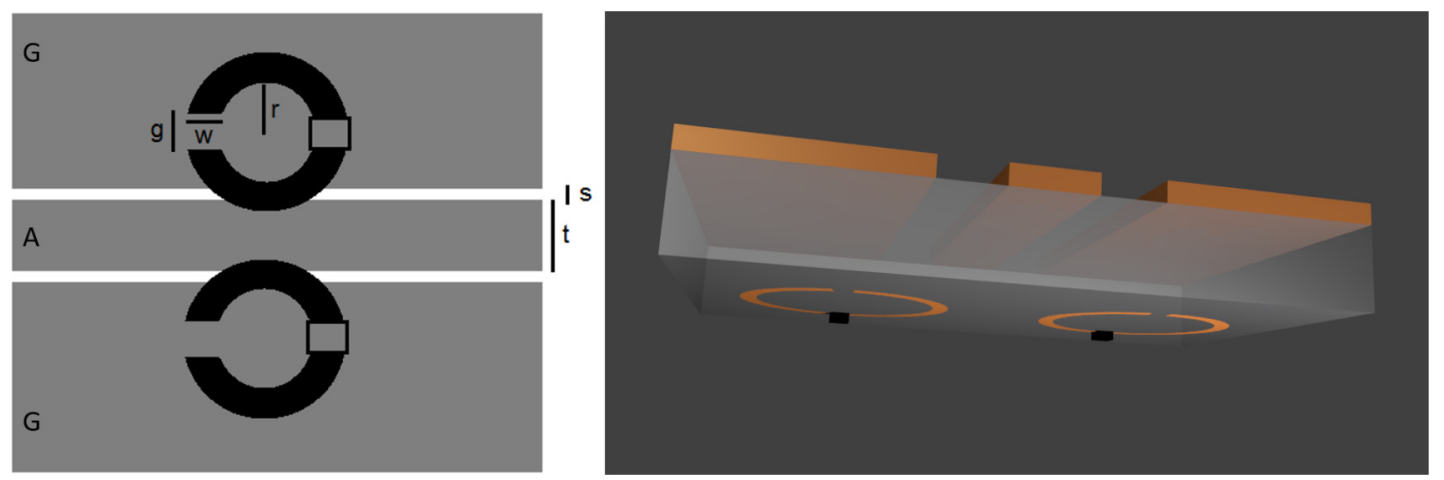

FIG. 2. Left: Schematic of a pair of active split-ring resonators (black) printed onto the reverse side of a coplanar waveguide (gray). The active component is located on the ring directly opposite the split. The distances marked are (not to scale): $g=0.1 \mathrm{~mm}, w=1 \mathrm{~mm}, r=3 \mathrm{~mm}$, $t=5 \mathrm{~mm}, s=0.5 \mathrm{~mm}$. The SRRs and CPW are separated by $1.6 \mathrm{~mm}$ of fiberglass substrate with a relative permittivity of 4.5 . Right: $3 \mathrm{D}$ schematic of active rings coupled to $\mathrm{CPW}$ 

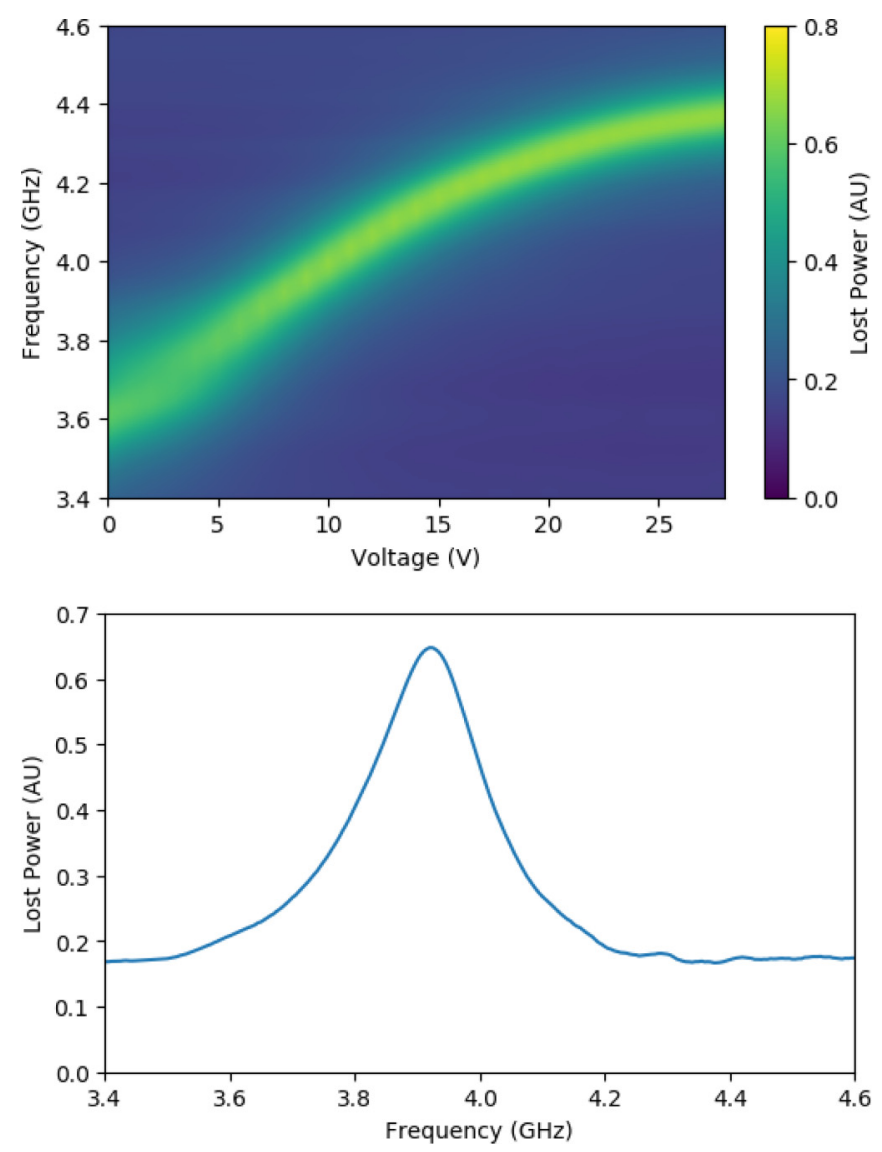

FIG. 3. Top: Experimentally measured transmitted intensity through a CPW coupled to SRRs loaded with variable capacitors (varactor) as a function of frequency and bias voltage across the varactor. The peak in loss, indicating a resonance of the SRR, is tuned from 3.61 to $4.46 \mathrm{GHz}$. Bottom: Cross section of the lost power at a bias voltage of $8 \mathrm{~V}$.

In order to characterize the response of the SRRs we measured the power reflected from, and transmitted through, a $3 \mathrm{~cm}$ length of our CPW + SRRs system as a function of frequency using an Anritsu MS46122B Vectorstar vector network analyzer (calibrated using a standard SOLT procedure). We subsequently calculated the power lost from the system, which is a combination of absorption and radiation scattered into free space, as 1-T-R. To characterize our tunable SRR we measured this lost power as the varactor was tuned across its full functional range of 0 to $28 \mathrm{~V}$ (see Fig. 3), yielding a tuning range of 3.61 to $4.36 \mathrm{GHz}$ for the resonance of the SRR.

\section{COUPLED SRRS}

Two split-ring resonators, when excited in close proximity to each other, exchange energy through their overlapping electric and magnetic fields. The pair of resonators act as coupled harmonic oscillators, allowing an analysis similar to that of a classical system of masses on springs [27]. For such a system of two coupled resonators there exist two hybridized modes; a higher frequency mode $\left(\omega_{+}\right)$and a lower frequency mode $\left(\omega_{-}\right)$. However, unlike simple mechanical resonators, since SRRs exhibit bianisotopy (an exciting electric field induces a magnetic dipole moment as well as an electric dipole moment and vice versa) one must consider the interaction between the SRRs via both the electric and magnetic dipole moments. From Fig. 1 one can see that the magnetic dipole moments of a pair of axially oriented SRRs will couple longitudinally, while the electric dipole moments will couple transversely. In addition, depending upon the relative rotation of the SRRs, the coupling via the electric and magnetic dipole moments can either be in conflict or in concert, allowing the strength of the coupling to be controlled via a rotation of one of the SRRs. A full description of the coupling mechanisms between axially oriented SRR dimers can be found in [8].

The complexity of these interactions requires an analysis similar to that of Liu et al. [28] where the modes of the two coupled rings can be derived from a Lagrangian analysis of the charges moving inside the rings. Equation (2) gives the frequency for the coupled modes $\omega_{+}$and $\omega_{-}$:

$$
\omega_{ \pm}^{2}=\frac{1}{2\left(1-K_{H}^{2}\right)}\left(\omega_{a}^{2}+\omega_{p}^{2}-2 K_{E} K_{H} \omega_{a} \omega_{p} \pm \sqrt{\left(\omega_{a}^{2}+\omega_{p}^{2}-2 K_{E} K_{H} \omega_{a} \omega_{p}\right)^{2}-4 \omega_{a}^{2} \omega_{p}^{2}\left(1-K_{H}^{2}\right)\left(1-K_{E}^{2}\right)}\right)
$$

where $\omega_{a}$ is the isolated resonance frequency of the tunable SRR, $\omega_{p}$ is the isolated resonance frequency of the passive SRR, and $K_{E}$ and $K_{H}$ are the dimensionless electrical and magnetic coupling terms, respectively. Equation (2) can be solved by treating $\omega_{+}$and $\omega_{-}$as simultaneous equations in order to find $K_{E}^{2}$ and $K_{H}^{2}$ in terms of the uncoupled frequencies.

\section{THE COUPLED MODES OF A PASSIVE AND TUNABLE SPLIT-RING RESONATOR PAIR}

To examine the coupled SRR interaction described in Sec. III we used a similar system to that described in Sec. II, but with the addition of a passive SRR placed between the CPW and the tunable SRR (see Fig. 4). The passive SRR was designed such that its resonance frequency was midway between the upper and lower frequency limits of the tunable SRR (approximately $4 \mathrm{GHz}$ ), thus allowing the resonance of the tunable SRR to be "tuned through" that of the passive SRR. To this end the passive SRR was designed with the following dimensions: $r=2.4 \mathrm{~mm}, g=0.8 \mathrm{~mm}, w=0.9 \mathrm{~mm}$.

While the tunable SRR was kept at a fixed distance from the $\mathrm{CPW}$, the separation between the tunable and nontunable SRRs could be altered through the use of additional spacer layers from 0.8 to $2.0 \mathrm{~mm}$ at $0.4 \mathrm{~mm}$ intervals. Altering this separation changes the coupling strength between the resonators since coupling arises from near-field interactions between the fields of the individual SRRs. In addition, the nontunable SRRs can be rotated relative to the tunable SRRs, giving an additional avenue for controlling the coupling strength. This rotation by angle $\theta$ is marked by the green arrow in Fig. 4. 


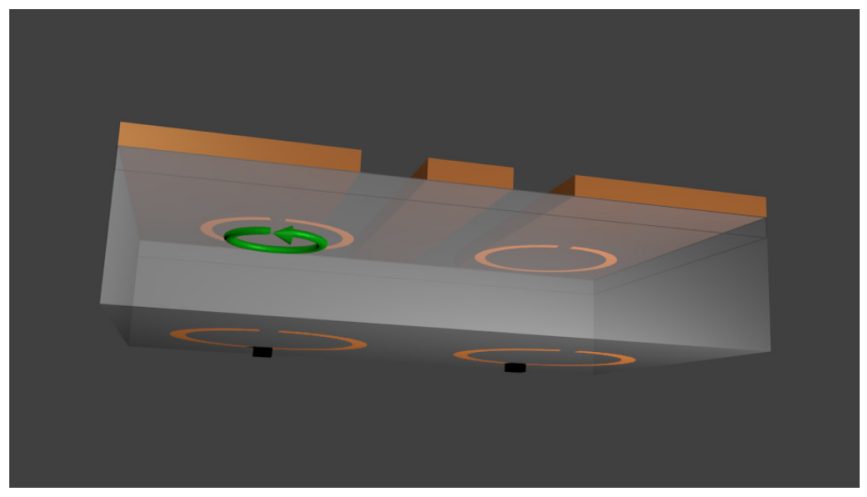

FIG. 4. 3D schematic of a CPW with split-ring resonators stacked beneath it. The lower rings are loaded with varactors (black regions) to make them actively tunable via an applied voltage. A pair of resonators is coupled to each track of the CPW to maintain symmetry. Shown in green is the angle of rotation $\theta$ used to reorientate the rings.

The loss from the system was measured to find the positions of $\omega_{+}$and $\omega_{-}$as a function of the tuning bias voltage for relative SRR rotations of $0^{\circ}, 90^{\circ}$, and $180^{\circ}$ and SRR separations of $0.8,1.2,1.6$, and $2.0 \mathrm{~mm}$. A plot of the loss spectra in the $90^{\circ}$ case is shown in Fig. 5. The mode positions were
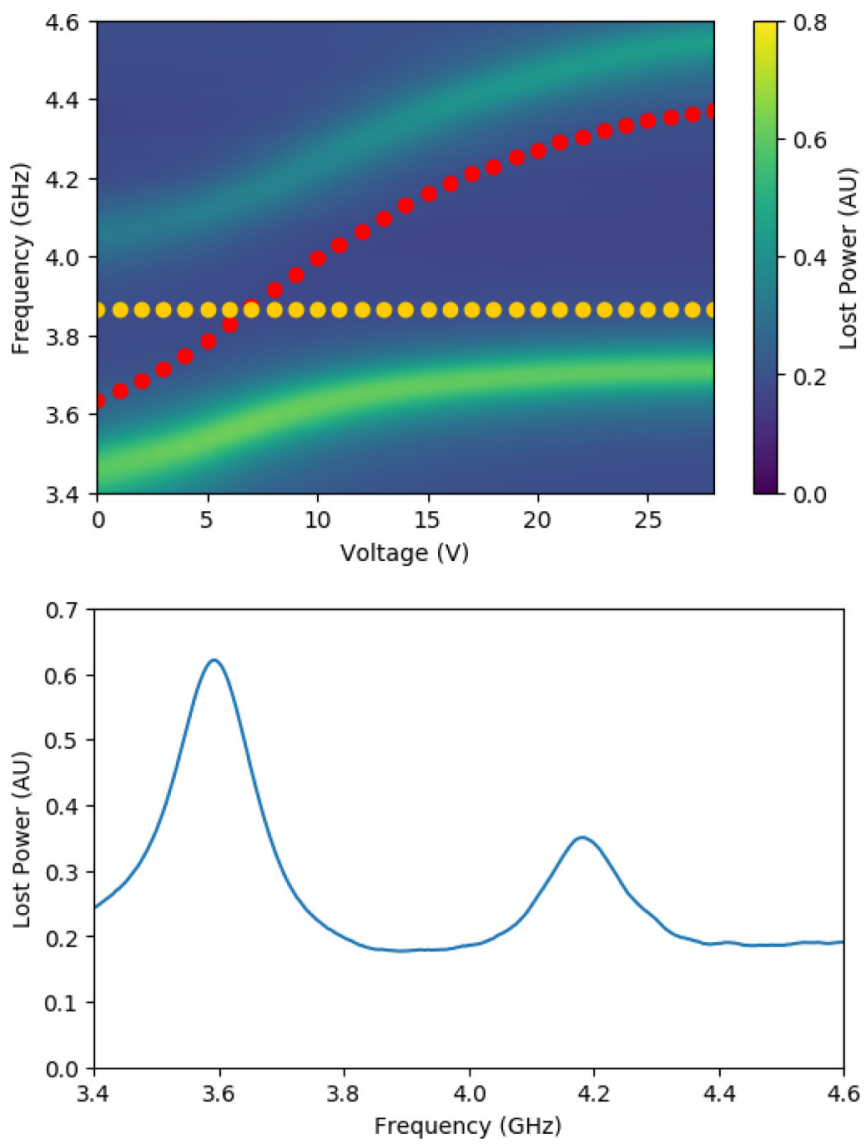

FIG. 5. Top: Plot of experimentally measured loss of the CPW coupled to an SRR pair as one ring is tuned through the resonant frequency of the other. The resonance frequencies of the individual active and passive SRRs are plotted as red and orange dotted lines, respectively. Bottom: Cross section of lost power at a bias voltage of $8 \mathrm{~V}$. extracted from these spectra using a peak fitting algorithm. In some cases the modes did not couple well to the CPW and were not directly observable in the loss spectra. In these cases estimates were made through a combination of matching to finite element models and keeping the calculated coupling terms continuous. Figure 6 shows theses extracted mode positions for all measured separations and angles.

The resonance frequency of the passive ring is slightly different for each data set as their orientation and proximity to the CPW has a small effect on their resonance frequency. Using the values for the mode positions extracted from the loss data sets, $K_{E}$ and $K_{H}$ where calculated, as described above, and are plotted in Fig. 6 using solid and dashed lines, respectively.

Starting with Figs. 6(a) and 6(d) we see that, in the $0^{\circ}$ case, while the frequency splitting between the modes is relatively small, the magnitude of the coupling terms, and hence the interaction strengths via the electric and magnetic dipole moments, is rather large. However, because the two coupling terms are in opposition with each other the net effect is a small splitting despite the strong interactions. Also notable is that $K_{E}$ and $K_{H}$ change which is of greatest magnitude as the tuning voltage is traversed for the $1.2,1.6$, and $2.0 \mathrm{~mm}$ separations. This indicates that by tuning the varactor one can determine whether the higher energy mode of the system is the symmetric or antisymmetric of the coupled modes.

In the $90^{\circ}$ case, Figs. 6(b) and 6(e), the frequency splitting is strong. However, the coupling appears to be dominated by $K_{E}$ while the $K_{H}$ term is minimal, despite the fact that the electric dipoles are orthogonal to each other. This is due to the rotational asymmetry of the ring; when the SRR is rotated the electric dipoles are not only rotated, they are also offset from each other. This allows for a strong electrical interaction between the dipoles where theories that only take account of the angle predict none [28]. Also notable in this case is the fact that the magnetic terms, while small, go through a transition near the uncoupled crossing point where they change from being in opposition to the electric terms to be being in concert with them.

Finally the $180^{\circ}$ case displays the greatest splitting of the three rotations, but has coupling terms that are no bigger, and if anything are smaller in some cases, than the others. The small values are because the $180^{\circ}$ case leads to the largest horizontal offset, and hence smallest interaction strength between the electric dipole moments, of the three rotations. However, this does not result in a smaller splitting because $K_{E}$ and $K_{H}$ work in concert for this orientation. Also notable in this plot is the crossing between $K_{E}$ and $K_{H}$ in the $0.8 \mathrm{~mm}$ separation case. Since $K_{E}$ and $K_{H}$ act in concert this does not result in a change in dipole symmetry of the modes, as was the case for $0^{\circ}$. Instead, whether the asymmetric splitting is weighted to be above or below the passive frequency, is governed by the relative magnitude of $K_{E}$ and $K_{H}$.

\section{CONCLUSION}

We have designed and fabricated a tunable split-ring resonator by incorporating a varactor. Through the application of a bias voltage the resonance frequency of the fundamental mode of the SRR can be tuned between 3.61 and $4.48 \mathrm{GHz}$. This range is 5 times larger than the frequency width of 
$0^{\circ}$

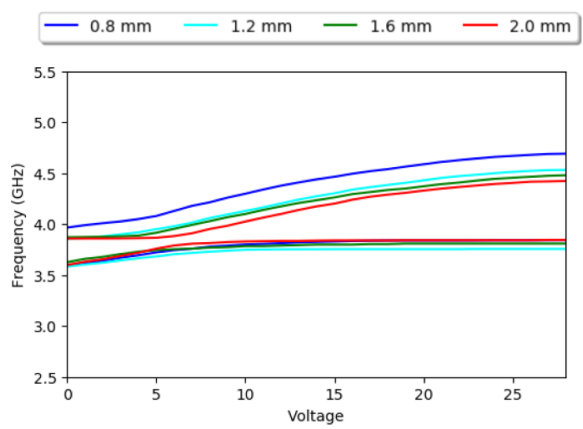

(a)

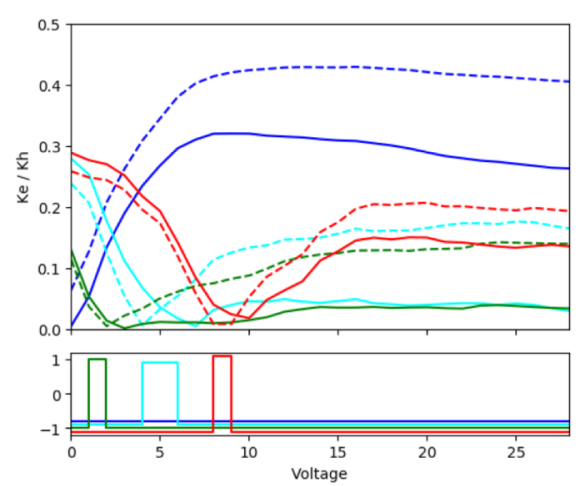

(d) $90^{\circ}$

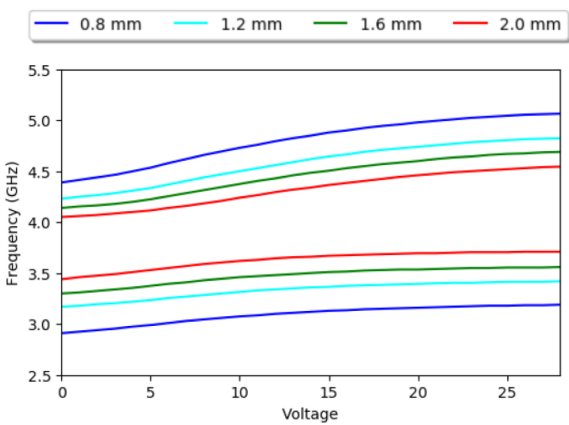

(b)

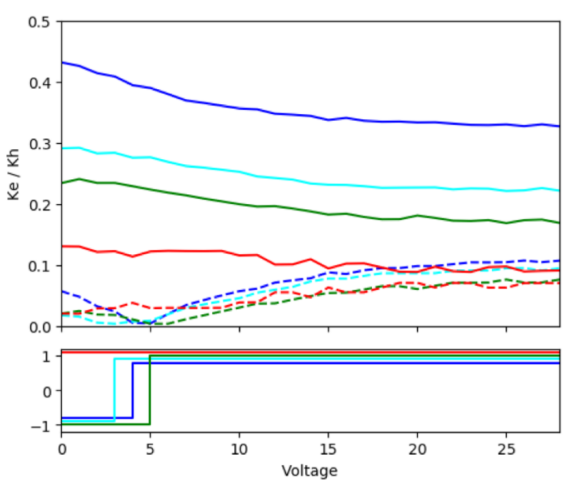

(e) $180^{\circ}$

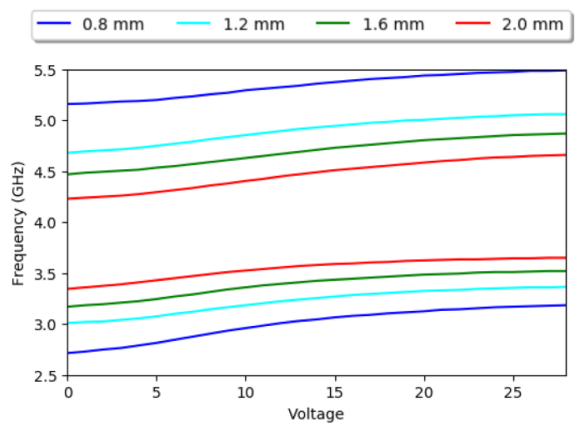

(c)

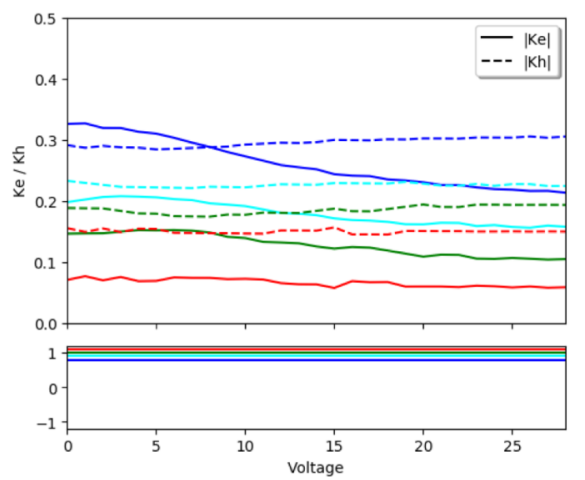

(f)

FIG. 6. Plots of upper and lower mode frequencies (a)-(c) and coupling terms $K_{E}$ (solid line) and $K_{H}$ (dashed line) (d)-(f) for systems of two coupled SRRs at separations $0.8,1.2,1.6$, and $2.0 \mathrm{~mm}$ (different colors) and relative orientations of $0^{\circ}$ (a) and (d), $90^{\circ}$ (b) and (e), and $180^{\circ}$ (c) and (f) as a function of the bias voltage applied to the varactors. The bottom segments of (d)-(f) show whether the electric and magnetic coupling terms are in conflict $(-1)$ or in concert (1) but have been slightly offset for legibility.

the mode (160 MHz). We have used this tunable SRR to investigate the coupling to a second nontunable SRR, and have observed an anticrossing behavior typical of coupled systems by tuning one ring's resonance frequency through that of the other. By changing the separation between the rings, and their relative rotation, we have been able to modify the strength of the coupling between the SRRs over a wide range. Such investigations into the coupling between metaatoms in close proximity to each other may help illuminate the difficulties and opportunities when designing dense active metamaterials.

\section{ACKNOWLEDGMENTS}

We acknowledge financial support from the Engineering and Physical Sciences Research Council (EPSRC) of the United Kingdom, via the EPSRC Centre for Doctoral Training in Metamaterials (Grant No. EP/L015331/1). W.L.B. acknowledges the support of the European Research Council through project Photmat (ERC-2016-AdG-742222). I.R.H. acknowledges support from the EPSRC via the TEAM-A prosperity partnership (Grant No. EP/L015331/1). We would also like to thank Sathya Sai Seetharaman for his help and advice.
[1] H. Lamb, On Group-Velocity, 1st ed. (C.F. Hodgson \& Son, London, 1904).

[2] W. J. Padilla, D. N. Basov, and D. R. Smith, Mater. Today 9, 28 (2006).

[3] J.-H. Lee, J. P. Singer, and E. L. Thomas, Adv. Mater. 24, 4782 (2012).

[4] K. Yao and Y. Liu, Nanotechnol. Rev. 3, 177 (2014).

[5] D. R. Smith, J. Gollub, J. J. Mock, W. J. Padilla, and D. Schurig, J. Appl. Phys. 100, 024507 (2006).

[6] X. Liu, H. Liu, Q. Sun, and N. Huang, Appl. Opt. 54, 3478 (2015).

[7] A. Ishikawa and T. Tanaka, Opt. Commun. 258, 300 (2006).
[8] S. S. Seetharaman, C. G. King, I. R. Hooper, and W. L. Barnes, Phys. Rev. B 96, 085426 (2017).

[9] H. Liu, D. A. Genov, D. M. Wu, Y. M. Liu, Z. W. Liu, C. Sun, S. N. Zhu, and X. Zhang, Phys. Rev. B 76, 073101 (2007).

[10] E. Shamonina, V. A. Kalinin, K. H. Ringhofer, and L. Solymar, J. Appl. Phys. 92, 6252 (2002).

[11] M. Decker, S. Linden, and M. Wegener, Opt. Lett. 34, 1579 (2009).

[12] N. Liu and H. Giessen, Opt. Express 16, 21233 (2008).

[13] I. V. Shadrivov, A. N. Reznik, and Y. S. Kivshar, Phys. B: Condens. Matter 394, 180 (2007). 
[14] A. Radkovskaya, O. Sydoruk, M. Shamonin, E. Shamonina, C. J. Stevens, G. Faulkner, D. J. Edwards, and L. Solymar, IET Microwav. Antennas Propag. 1, 80 (2007).

[15] I. Sersic, M. Frimmer, E. Verhagen, and A. F. Koenderink, Phys. Rev. Lett. 103, 213902 (2009).

[16] M. Lapine, D. Powell, M. Gorkunov, I. Shadrivov, R. Marqués, and Y. Kivshar, Appl. Phys. Lett. 95, 084105 (2009).

[17] A. A. Oliner, IEEE MTT-S Int. Microw. Symp. Digest 1, 191 (2003).

[18] N. Kundtz and D. R. Smith, Nat. Mater. 9, 129 (2010).

[19] D. A. Powell, M. Lapine, M. V. Gorkunov, I. V. Shadrivov, and Y. S. Kivshar, Phys. Rev. B 82, 155128 (2010).

[20] D. A. Powell, K. Hannam, I. V. Shadrivov, and Y. S. Kivshar, Phys. Rev. B 83, 235420 (2011).
[21] M. Kang, F. Liu, and J. Li, Phys. Rev. A 87, 053824 (2013).

[22] J. Schindler, A. Li, M. C. Zheng, F. M. Ellis, and T. Kottos, Phys. Rev. A 84, 040101(R) (2011).

[23] O. Sydoruk, E. Tatartschuk, E. Shamonina, and L. Solymar, J. Appl. Phys. 105, 014903 (2009).

[24] X. Wang, P. Bao, and T. J. J. M. J. Lancaster, IET Microw. Antennas Propag. 5, 776 (2011).

[25] J. D. Baena, J. Bonache, F. Martín, R. M. Sillero, F. Falcone, T. Lopetegi, M. A. G. Laso, J. G. García, I. Gil, M. F. Portillo, and M. Sorolla, IEEE Trans. Microw. Theory Tech. 53, 1451 (2005).

[26] D. M. Pozar, Microwave Engineering (Wiley, New York, 2004), pp. 141-147.

[27] L. Novotny, Am. J. Phys. 78, 1199 (2010).

[28] T. Q. Li, H. Liu, T. Li, S. M. Wang, J. X. Cao, Z. H. Zhu, Z. G. Dong, S. N. Zhu, and X. Zhang, Phys. Rev. B 80, 115113 (2009). 